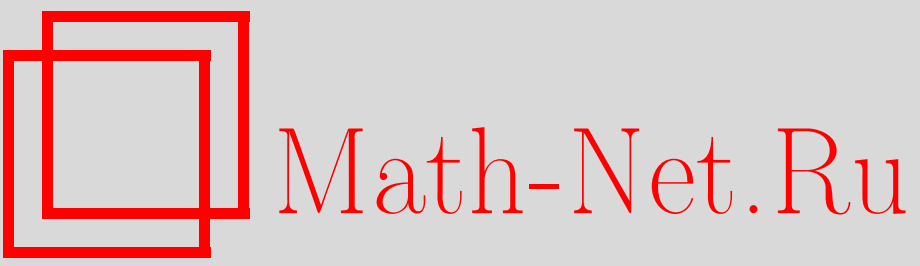

С. А. Степин, Особые точки дифференциальных уравнений второго порядка: решения с логарифмическими членами, УМН, 1999, том 54, выпуск 1, 265-266

DOI: https://doi.org/10.4213/rm130

Использование Общероссийского математического портала Math-Net.Ru подразумевает, что вы прочитали и согласны с пользовательским соглашением

http://www.mathnet.ru/rus/agreement

Параметры загрузки:

IP: 34.239 .49 .27

26 апреля 2023 г., 15:12:57 


\title{
ОСОБЫЕ ТОЧКИ ДИФФЕРЕНЦИАЛЬНЫХ УРАВНЕНИЙ ВТОРОГО ПОРЯДКА: РЕШЕНИЯ С ЛОГАРИФМИЧЕСКИМИ ЧЛЕНАМИ
}

\author{
С. А. Степин
}

Особая точка линейного обькновенного дифференциального уравнения может оказаться для его решений точкой ветвления конечного порядка, либо логарифмической точкой ветвления. В настоящей работе рассматривается уравнение второго порядка

$$
z^{2} \frac{d^{2} w}{d z^{2}}+z a(z) \frac{d w}{d z}+b(z) w=0
$$

для которого $z=0$ является регулярной особой точкой, т.е. коэффициенты $a(z)$ и $b(z)$ аналитичны в окрестности $z=0$, причем одно из чисел $a(0), b(0), b^{\prime}(0)$ отлично от 0 .

Пусть $\lambda_{1}, \lambda_{2}$ - корни определяющего уравнения

$$
\lambda^{2}+(a(0)-1) \lambda+b(0)=0
$$

$\operatorname{Re} \lambda_{1} \geqslant \operatorname{Re} \lambda_{2}$. Известно (см., например, [1]), что при условии $\lambda_{1}-\lambda_{2} \notin \mathbb{Z}$, уравнение (1) имеет фундаментальную систему решений $(\Phi \mathrm{CP})$ вида

$$
w_{1}(z)=z^{\lambda_{1}} \varphi(z), \quad w_{2}(z)=z^{\lambda_{2}} \psi(z),
$$

где $\varphi(z), \psi(z)$ аналитичны в некоторой окрестности точки $z=0$. Если же $\lambda_{1}-\lambda_{2} \in \mathbb{Z}$, то $\Phi С Р$ уравнения (1) имеет вид

$$
w_{1}(z)=z^{\lambda_{1}} \varphi(z), \quad w_{2}(z)=z^{\lambda_{2}} \psi(z)+C w_{1}(z) \ln z,
$$

причем постоянная $C$ может "случайно" оказаться нулем и тогда $\Phi С Р$ выглядит так же как и в случае $\lambda_{1}-\lambda_{2} \notin \mathbb{Z}$.

Постановка и иследование вопроса о структуре решений обыкновенного дифференциального уравнения в окрестности регулярной особой точки восходят к работам Фробениуса и Фукса (см. [2; гл. 16]). Обзор результатов об условиях отсутствия логарифмических членов в $\Phi С Р$ имеется в [3]; см. также [4]. Ниже в терминах $a(z)$ и $b(z)$ найдено явное выражение для коэффициента $C$ при логарифмическом слагаемом решения $w_{2}(z)$ в резонансном случае $\lambda_{1}-\lambda_{2} \in \mathbb{N}$.

Предварительно с помощью замены

$$
w(z)=y(z) \exp \left(-\frac{1}{2} \int \frac{a(z)}{z} d z\right)
$$

уравнение (1) преобразуется к виду

$$
z^{2} \frac{d^{2} y}{d z^{2}}+q(z) y=0
$$

где $q(z)=b(z)-a^{2}(z) / 4+\left(a(z)-z a^{\prime}(z)\right) / 2$ - аналитическая в окрестности $z=0$ функция: $q(z)=\sum_{k=0}^{\infty} q_{k} z^{k}$. Структура ФСР уравнения (3) в окрестности $z=0$ такая же, как и у (1), причем характеристические показатели $\mu_{1}, \mu_{2}$ преобразованного уравнения равны соответственно $\lambda_{1}+a(0) / 2$ и $\lambda_{2}+a(0) / 2$. Введем обозначение $F_{k}=k\left(k+\lambda_{2}-\lambda_{1}\right), k \in \mathbb{N}$.

Теорема. Пусть разность $\lambda_{1}-\lambda_{2}=n \in \mathbb{N}$. Тогда уравнение (1) имеет ФСР вида (2), əде $\varphi(0)=\psi(0)=1 u$

$$
\begin{aligned}
C=\frac{1}{n}\left\{-q_{n}\right. & +\sum_{k=1}^{n-1} \frac{q_{n-k} q_{k}}{F_{k}}-\sum_{k_{2}<k_{1}} \frac{q_{n-k_{1}} q_{k_{1}-k_{2}} q_{k_{2}}}{F_{k_{1}} F_{k_{2}}} \\
& +\cdots+(-1)^{j+1} \sum_{k_{j}<\cdots<k_{1}} \frac{q_{n-k_{1}} q_{k_{1}-k_{2}} \cdots q_{k_{j}}}{F_{k_{1}} F_{k_{2}} \cdots F_{k_{j}}} \\
& \left.+\cdots+(-1)^{n-1} \frac{q_{1}^{n-1} q_{2}}{F_{n-1} \cdots F_{1}} \sum_{k=1}^{n-1} F_{k}+(-1)^{n} \frac{q_{1}^{n}}{F_{n-1} \cdots F_{1}}\right\} .
\end{aligned}
$$


Пусть решение $y_{1}(z)$ уравнения (2), отвечающее характеристическому показателю $\mu_{1}$, нормировано условием $\lim _{z \rightarrow 0} y_{1}(z) / z^{\mu_{1}}=1$. Второе решение ищется в виде

$$
y_{2}(z)=z^{\mu_{2}}\left[1+\sum_{k=1}^{\infty} c_{k} z^{k}\right]+C y_{1}(z) \ln z .
$$

Подставляя это выражение в уравнение (3) и, приравнивая коэффициенты при одинаковых степенях переменной $z$, последовательно находим все $c_{k}$ и $C$. Введем матрицу $T=\left(t_{i j}\right)_{i, j=1}^{n-1}$, где $t_{i j}=0$ при $i+j<n, t_{i j}=F_{i}$, если $i+j=n$ и $t_{i j}=q_{i+j-n}$ для остальных $i, j$. Положим $v=\left(q_{1}, \ldots, q_{n-1}\right)$, тогда

$$
\begin{aligned}
C & =-\frac{1}{n}\left\{q_{n}+\left(T^{-1} v, v\right)\right\} \\
& =-\frac{1}{n}\left\{q_{n}-\sum_{k=1}^{n-1} \frac{q_{n-k} q_{k}}{F_{k}}+\sum_{j=2}^{n-1}(-1)^{j} \sum_{k_{j}<\cdots<k_{1}} \frac{q_{n-k_{1}} q_{k_{1}-k_{2}} \cdots q_{k_{j}}}{F_{k_{1}} F_{k_{2}} \cdots F_{k_{j}}}\right\} .
\end{aligned}
$$

Для того, чтобы решение $y_{2}(z)$ не содержало слагаемого с логариффмом, необходимым и достаточным является условие

$$
q_{n}-\sum_{k=1}^{n-1} \frac{q_{n-k} q_{k}}{F_{k}}+\sum_{j=2}^{n-1}(-1)^{j} \sum_{k_{j}<\cdots<k_{1}} \frac{q_{n-k_{1}} q_{k_{1}-k_{2}} \cdots q_{k_{j}}}{F_{k_{1}} F_{k_{2}} \cdots F_{k_{j}}}=0 .
$$

В этом случае решение $y_{2}(z)$ содержит произвольную постоянную $c_{n}$, а соответствующее слагаемое, у которого $c_{n}$ будет множителем, пропорционально $y_{1}(z)$ (см. [1]); без потери общности (с точки зрения построения $\Phi \mathrm{CP}$ ) можно положить $c_{n}=0$.

СлЕДствиЕ. Общее решение уравнения (1) имеет в точке $z=0$ не более чем алгебраическую особенность тогда и только тогда, когда характеристические показатели $\lambda_{1}, \lambda_{2} \in \mathbb{Q}, \lambda_{1} \neq \lambda_{2}$ и, кроме того, должно быть выполнено условие (4), если $\lambda_{1}-\lambda_{2}=n \in \mathbb{N}$.

Уравнение (1) можно свести к системе

$$
z \frac{d Y}{d z}=A(z) Y
$$

где $A(z)$ - матрица второго порядка, аналитическая в окрестности точки $z=0$. Согласно общей теории (см. [5]), такая система имеет фундаментальное матричное решение вида

$$
Y=P(z) \cdot z^{G}
$$

где $P(z)$ аналитична в окрестности $z=0$, а разность собственных значений постоянной матрицы $G$ не является положительным целым числом. Применительно к рассматриваемой ситуации условие (4) указывает те и только те случаи, когда матрица $G$ будет скалярной.

Работа выполнена при поддержке Российского фонда фундаментальных исследований.

\section{СПИСОК ЛИТЕРАТУРЫ}

[1] Уиттекер Э.Т., Ватсон Дж. Н. Курс современного анализа. Т. 1. М.: Физматгиз, 1963. [2] Айнс Э. Л. Обыкновенные диференциальные уравнения. Харьков: ГНТИ Украины, 1939. [3] Harris W. A. // Appl. Anal. 1978. V. 8. P. 171-174. [4] Григоренко Н. В. // Матем. заметки. 1983. Т. 33. №6. С. 881-884. [5] Вазов В. Асимптотические разложения решений обыкновенных дифференциальных уравнений. М.: Мир, 1968. 